\title{
Relative bioavailability and comparative clinical efficacy of different ivermectin oral formulations in lambs
}

Gonzalo Suárez ${ }^{1}$, Luis Alvarez ${ }^{2}$, Daniel Castells ${ }^{3}$, Oscar Correa ${ }^{4}$, Pietro Fagiolino ${ }^{5}$ and Carlos Lanusse $2^{2^{*}}$

\begin{abstract}
Background: Several oral ivermectin (IVM) formulations for use in sheep are available in the pharmaceutical veterinary market in different countries. All of them are indicated at the same dose rate to treat the gastrointestinal nematodes. However, there is a lack of information on the relative systemic exposure (plasma bioavailability) and clinical efficacy among oral formulations routinely used in sheep. The main goal of the work reported here was to perform a pharmaco-parasitological assessment of three different IVM oral formulations in lambs infected with multiple resistant gastrointestinal nematodes. The comparative drug systemic exposure (IVM plasma concentrations) and nematodicidal efficacies (clinical efficacy) in lambs were determined for a reference (RF) and two different test $(T 1, T 2)$ IVM oral formulations. One hundred and fifty six $(n=156)$ healthy Corriedale lambs, naturally infected with multiple resistant gastrointestinal nematodes were allocated into four experimental groups $(n=39)$. Animals in each group received treatment $(200 \mathrm{\mu g} / \mathrm{kg})$ with either the $\mathrm{RF}$, one of the test IVM formulations or were kept as untreated control. Blood samples were collected over 15 days post-treatment $(n=8)$. The IVM plasma concentrations were measured by high performance liquid chromatography with fluorescence detection. The faecal nematode egg count reduction test (FECRT) $(n=39)$ and evaluation of the clinical efficacy were performed at day 14 post-treatment $(n=6)$, where a predominance of IVM highly resistant nematodes was observed.
\end{abstract}

Results and conclusions: Neither the overall kinetic behaviour nor the IVM systemic exposure differed among all the tested oral formulations. Equivalent efficacy results were obtained for the different preparations, with an evident therapeutic failure to control Haemonchus spp. and Teladorsagia circumcincta, which correlates with a high degree of nematode resistance to IVM.

\section{Background}

Ivermectin (IVM), a member of the macrocyclic lactone antiparasitic drugs, exhibits a broad-spectrum of activity against gastrointestinal (GI) and lung nematodes [1] as well as against ectoparasites of clinical relevance in domestic animals [2,3]. In sheep and goats at the dose of $0.2 \mathrm{mg} / \mathrm{kg}$, IVM efficacy claims included Haemonchus spp., Teladorsagia circumcincta, Ostertagia trifurcata, Trichostrongylus spp., Nematodirus spp., Cooperia spp., Oesophagostomum spp., Chavertia ovina and Trichuris ovis, among the most important nematodes [4].

\footnotetext{
* Correspondence: clanusse@vet.unicen.edu.ar

${ }^{2}$ Laboratorio de Farmacología, Centro de Investigación Veterinaria de Tandil (CIVETAN), CONICET, Facultad de Ciencias Veterinarias, Universidad Nacional del Centro de la Provincia de Buenos Aires, Campus Universitario, (7000) Tandil, Argentina

Full list of author information is available at the end of the article
}

Additionally, its extensive tissue distribution, low biotransformation and high plasma-gastrointestinal (GI) recycling assure its persistent activity. Consequently, IVM is the most widely used anthelmintic, and this extensive use has led to the selection and emergence of IVM-resistant nematode populations in several areas of the world [5]. This is particularly relevant taking into consideration the rapid spread of parasite resistance in sheep nematodes.

In Uruguay, the registration of a "new" anthelmintic formulation is only based in a field efficacy study; information related to the pharmacokinetic behaviour of the specific formulation is not required. Several oral IVM formulations for use in lambs are available in the pharmaceutical veterinary market in Uruguay. All of them are indicated at the same dose rate $(0.2 \mathrm{mg} / \mathrm{kg})$ to 
treat GI nematodes. However, the route of administration and the formulation type strongly affect IVM plasma pharmacokinetic behaviour [6,7]. Differential systemic exposures were observed in cattle after the subcutaneous administration of IVM formulated as different commercial formulations $[7,8]$. Furthermore, some drastic pharmacokinetic differences were observed among generic albendazole formulations available for use in sheep $[9,10]$. However, there is a lack of information on the relative bioavailability among oral IVM formulations in sheep. Additionally, the impact on clinical efficacy against either dose-limiting or resistant nematodes related to drug-absorption differences due to the type and/or quality of pharmaceutical preparation needs to be addressed.

Bioequivalence/Relative bioavailability of a given anthelmintic drug should serve as additional evidence of equivalence in activity [11]. The estimation of the relative bioavailability is useful to compare the extent of absorption of different drug formulations of the same active ingredient. Assuming that a relationship exists between plasma concentration of the active moiety and clinical efficacy, knowledge of the bioavailability and disposition kinetics of the active compound would be particularly useful in the development of dosage forms and for comparison of routes of administration/formulations [12].

The mail goals of the current work were: 1) to determine the comparative IVM systemic exposure (relative bioavailability) obtained after treatment with three different oral formulations available in Uruguay for use in sheep, and 2) to investigate the efficacy of the three preparations against IVM resistant nematode parasites.

\section{Results}

Analytical procedures, including chemical extraction, derivatization and HPLC analysis of IVM in lamb plasma were appropriately validated. The linear regression lines for IVM in plasma in the range $0.1-2.0 \mathrm{ng} / \mathrm{mL}$ and $2.0-40 \mathrm{ng} / \mathrm{mL}$ showed correlation coefficients from 0.9994 to 0.9972 and the departure from linearity was not statistically significant. The intra and inter assay precision of the analytical procedures obtained after HPLC analysis of IVM on different working days showed CV $3.54 \%$ and $4.25 \%$, respectively. The LOQ was established at $0.1 \mathrm{ng} / \mathrm{mL}$.

Figure 1 depicts the mean $( \pm S D)$ IVM plasma concentration profiles obtained following the intraruminal (i.r.) administration of the RF (pioneer product) and each of the test generic (T1 and T2) IVM commercial formulation in parasitized lambs. Table 1 summarizes the main pharmacokinetic parameters obtained after the administration of IVM as the different assayed commercial formulations. IVM was first detected in plasma between $1 \mathrm{~h}$ and 6 days post-administration. The overall disposition kinetic of IVM was similar following treatment with each formulation. No

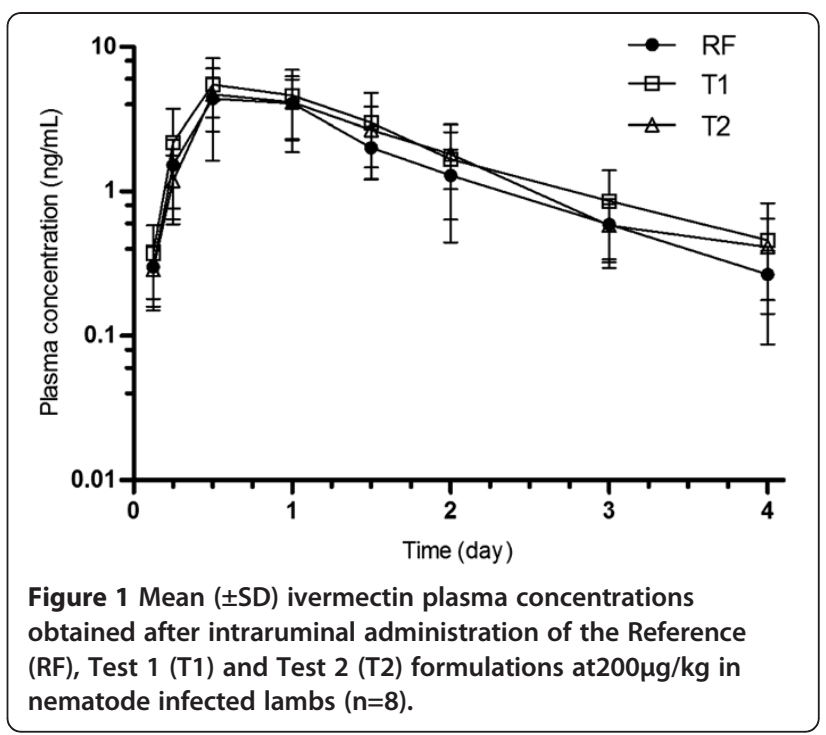

statistical differences among formulations were observed in the different pharmacokinetic variables, including those related to IVM distribution $\left(\mathrm{Vd}_{\text {area }} / \mathrm{F}\right)$ and elimination (MRT, $\mathrm{T}_{1 / 2 \mathrm{e}}, \mathrm{Cl}_{\star} / \mathrm{F}$ ) patterns (Table 1 ). IVM relative bioavailability was 132 and $117 \%$ for T1 and T2 formulation, respectively.

The mean $( \pm \mathrm{SD})$ eggs per gram of faeces (epg) counts at 14 days after treatment for RF, T1, T2 and untreated control $(\mathrm{n}=39)$ were $5029( \pm 3673) ; 5100 \quad( \pm 3817) ; 5481$ $( \pm 3831)$ and $5413( \pm 4349)$, respectively. Haemonchus spp.

Table 1 Mean ( \pm SD) ivermectin pharmacokinetic parameters obtained after the intraruminal administration of the Reference (RF), Test 1 (T1) and Test 2 (T2) formulations at $200 \mu \mathrm{g} / \mathrm{kg}$ in nematode infected lambs $(\mathbf{n}=\mathbf{8})$

\begin{tabular}{|c|c|c|c|c|}
\hline Parameters & Reference & Test 1 & Test 2 & $P$ value \\
\hline$C_{\max }(n g / m L)$ & $5.14 \pm 2.46$ & $5.82 \pm 2.53$ & $4.96 \pm 1.21$ & 0.776 \\
\hline $\mathrm{T}_{\max }$ (days) & $0.81 \pm 0.26$ & $0.71 \pm 0.27$ & $0.60 \pm 0.22$ & 0.341 \\
\hline$A \cup C_{0-L O Q}$ (ng.days/mL) & $6.92 \pm 3.26$ & $9.18 \pm 5.20$ & $8.11 \pm 3.27$ & 0.568 \\
\hline$A \cup C_{0-\infty}$ (ng.days $\left./ m L\right)$ & $7.20 \pm 3.23$ & $9.39 \pm 5.20$ & $8.35 \pm 3.29$ & 0.588 \\
\hline$A \cup C_{0-L O Q} / A \cup C_{0-\infty}$ & 0.96 [3.9\%] & $0.98[2.3 \%]$ & $0.97[2.9 \%]$ & - \\
\hline MRT (days) & $1.61 \pm 0.24$ & $1.60 \pm 0.40$ & $1.75 \pm 0.39$ & 0.724 \\
\hline $\mathrm{T}_{1 / 2 \mathrm{el}}$ (days) & $1.07 \pm 0.35$ & $0.90 \pm 0.21$ & $1.12 \pm 0.45$ & 0.492 \\
\hline$C_{\max } / A \cup C_{0-L O Q}$ & $0.74 \pm 0.13$ & $0.67 \pm 0.22$ & $0.64 \pm 0.12$ & 0.542 \\
\hline $\mathrm{CL}_{\Lambda} / \mathrm{F}$ (L/days) & $32.3 \pm 12.2$ & $25.6 \pm 10.2$ & $27.3 \pm 10.9$ & 0.505 \\
\hline $\mathrm{Vd}_{\text {area }} / \mathrm{F}$ (L/days) & $48.5 \pm 19.5$ & $32.4 \pm 13.5$ & $40.5 \pm 13.2$ & 0.189 \\
\hline
\end{tabular}

$C_{\text {max }}$ : peak plasma concentration; $T_{\text {max }}$ t time to peak plasma concentration; ${ }_{A \cup C} C_{0-\mathrm{LOQ}}$ area under the concentration vs. time curve form 0 up to the limit of quantification; $\mathrm{AUCO}_{-\infty}$ : area under the concentration vs. time curve extrapolated to infinity; MRT: mean residence time; $\mathrm{T}_{1 / 2 \mathrm{el}}$ : elimination half-life. $\mathrm{CL}_{\lambda} / \mathrm{F}$ : apparent total body clearance; $\mathrm{Vd}_{\text {area }} / \mathrm{F}$ : apparent volume of distribution (area method). $V d_{\text {area }}$ and $C L_{\lambda}$ represent their true values divided by the systemic availability (F) of either drug. In values within a row no statistical differences were observed $(P>0.05)$. The percentage that $A U C_{0-L O Q}$ differs from $A U C_{0-\infty}$ is reported in bracket []. 
represented $99-100 \%$ of the total $\mathrm{L}_{3}$ recovered from fecal cultures in all group. The adult nematode counts and the efficacy results obtained for the different treatments are shown in Table 2. Upon necropsy, worms were recovered from all the IVM treated groups. The Haemonchus spp. genus resulted to be the most prevalent in the untreated control group. Besides, T. circumcincta, Trichostrongylus spp., Nematodirus spp., Cooperia spp., Oesophagostomum spp. and Trichuris ovis were recovered in a lower number.

In this field study, the indirect efficacy estimated by means of the FECRT showed a low percentage of reduction for the RF (7.1\%) as well as for the T1 (5.8\%) and T2 $(0 \%)$ formulation in comparison to the untreated control $(P>0.05)$. The efficacy of all the formulations demonstrated that Haemonchus spp. was basically refractory to the IVM treatment $(<27 \%)$. The worm count data are in concordance with the FECRT and larval differentiation data in fecal cultures. Low efficacy $(<80 \%)$ against T. circumcincta, Cooperia spp. and Nematodirus spp. was observed in the IVM treated groups. In contrast, IVM demonstrated to be highly efficacious against Trichostrongylus spp. in abomasum and small intestine. Due to variations in individual Trichostrongylus spp. counts, the observed differences did not reach statistical significance $(\mathrm{P}>0.05)$ compared to the untreated control in abomasums (RF and T2) and small intestine (T1). In all treated groups, no worms were recovered from the large intestine.

\section{Discussion}

The macrocyclic lactones are the most widely used broad-spectrum antiparasitic drugs in veterinary medicine. Their notorious popularity is related to a high efficacy against ecto and endo parasites (nematodes), high potency, persistent activity and low toxicity. IVM, the first commercially available macrocyclic lactone endectocide, was introduced in the pharmaceutical market in the early ' 80s. Since the IVM patent protection expire, several "similar" (generic) products entered the veterinary market worldwide. Uruguay was not an exception, according official data more than 60 different IVM formulations are currently registered for use in veterinary medicine, from which thirteen are solutions for oral administration to be used in sheep. The large number of available commercial formulations, situation that is reflected in many other countries around the world, faces the problem of a lack of information on their absorption patterns, which seems to be critical considering

Table 2 Mean number of worms (range) and efficacy (\%) from necropsy performed 14 days after the intraruminal administration of the reference (RF) and each of test generic (Test 1 and Test 2) ivermectin formulations at $200 \mu \mathrm{g} / \mathrm{kg}$ in nematode infected lambs $(n=6)$

\begin{tabular}{|c|c|c|c|c|c|c|c|}
\hline \multirow[t]{2}{*}{ Parasites } & \multicolumn{2}{|c|}{ Reference } & \multicolumn{2}{|c|}{ Test 1} & \multicolumn{2}{|c|}{ Test 2} & \multirow{2}{*}{$\begin{array}{l}\text { Untreated control } \\
\text { Worm counts }\end{array}$} \\
\hline & Worm counts & Efficacy (\%) & Worm counts & Efficacy (\%) & Worm counts & Efficacy (\%) & \\
\hline \multicolumn{8}{|l|}{ Abomasum } \\
\hline \multirow[t]{2}{*}{ Haemonchus spp. } & 3210 & 7 & 3117 & 8 & 2514 & 27 & 3362 \\
\hline & $(2190-4430)$ & & $(1920-3850)$ & & $(1591-3950)$ & & $(2680-4360)$ \\
\hline \multirow[t]{2}{*}{ Teladorsagia circumcincta } & 292 & 39 & 189 & 57 & 272 & 44 & 520 \\
\hline & $(90-530)$ & & $(100-310)$ & & $(680-130)$ & & $(240-1460)$ \\
\hline \multirow[t]{2}{*}{ Trichostrongylus axei } & 73 & 96 & $47 *$ & 96 & 140 & 97 & 375 \\
\hline & $(0-220)$ & & $(0-160)$ & & $(0-440)$ & & $(80-1160)$ \\
\hline \multicolumn{8}{|l|}{ Small intestine } \\
\hline \multirow[t]{2}{*}{ Trichostrongylus columbriformis } & $10 *$ & 100 & 20 & 94 & $3 *$ & 100 & 140 \\
\hline & $(0-40)$ & & $(0-50)$ & & $(0-10)$ & & $(10-380)$ \\
\hline \multirow[t]{2}{*}{ Cooperia spp. } & 40 & 80 & 28 & 38 & 17 & 61 & 70 \\
\hline & $(0-150)$ & & $(0-50)$ & & $(0-40)$ & & $(0-190)$ \\
\hline \multirow[t]{2}{*}{ Nematodirus spp. } & 25 & 83 & 38 & 78 & $5 *$ & 100 & 278 \\
\hline & $(0-60)$ & & $(0-90)$ & & $(0-30)$ & & $(0-980)$ \\
\hline \multicolumn{8}{|l|}{ Large intestine } \\
\hline \multirow[t]{2}{*}{ Oesophagostomun spp. } & $0 *$ & 100 & $0 *$ & 100 & $0 *$ & 100 & 16 \\
\hline & $(0-0)$ & & $(0-1)$ & & $(0-0)$ & & $(2-19)$ \\
\hline \multirow[t]{2}{*}{ Trichuris ovis } & $0 *$ & 100 & $0 *$ & 100 & $0 *$ & 100 & 5 \\
\hline & $(0-0)$ & & $(0-0)$ & & $(0-0)$ & & $(2-10)$ \\
\hline
\end{tabular}

The percentage of efficacy was calculated using geometric mean as suggested by Wood et al. (1995).

* Nematode counts are statistically different $(P<0.05)$ compared to counts obtained in the untreated control group. 
the possibility of differences on manufacturing processes and quality of components that may exist among formulations. These differences may substantially affect drug dissolution and its consequent GI absorption, which in turns could affect drug effectiveness. The comparison of the systemic drug exposure (measured as plasma concentration profiles) after treatment with different IVM generic formulation is an initial approach to check their pharmacotechnical quality, which has been shown to drastically affect the systemic availability of other active ingredients (i.e. albendazole) [10]. A RF and two generic IVM preparations were selected to be tested in the work reported here. The selection of the formulations did not respond to any particular interest to compare the quality among them. However, the comparison was done in order to simulate a real practical situation that could result useful to illustrate a market situation with a great impact on parasite control.

In order to assess the pharmacokinetic behaviour of different formulations, absorption related pharmacokinetic parameters must primarily be considered. The AUC, which reflects the extent to which the active drug is absorbed and is independent of the rate of the absorption process, and $C_{\max }$, which indicates the extent and the rate of drug absorption. Since differences in body condition, breed, gender, feeding, and parasitism substantially affect the plasma disposition kinetics of macrocyclic lactones (reviewed by [13]), the current study was conducted in lambs with similar characteristics, uniformly distributed among experimental groups. This is particularly important for studies conducted using a parallel design, since this experimental design has a lower power than the cross-over design for relative bioavailability [14]. However, the use of a parallel design can provide useful information on gross deficiencies in the absorption process of different anthelmintic formulations [10]. Similar ( $P>0.05)$ IVM plasma AUC and Cmax (Table 1) were observed among formulations, suggesting a similar extent of absorption among the addressed reference and generic formulations. Tmax and Cmax/AUC did not show significant differences among the studied formulations, revealing a similar rate of the absorption process. Furthermore, our study showed similar values for other pharmacokinetic parameters (Table 1). Since a similar pharmacokinetic behavior was observed for IVM after the administration of the RF compared to both test formulations in animals grazed on pasture, it could be concluded that the assayed commercial preparations deliver IVM in an equivalent way which may indicated that manufacturing and overall pharmaceutical quality did not differ among them.

A lower IVM plasma drug exposure (expressed as Cmax and AUC) was observed in the current experiment, compared to that previously reported [15]. This may be related to differences in some experimentrelated factors (parasitism, breed, body condition, feed, etc.) which have shown to affect the pharmacokinetic behaviour of IVM [13]. On the other hand and as it was previously reported, IVM plasma concentrations is higher after the SC compared to the IR administration $[16,17]$. Although similar concentration profiles were measured in the abomasal mucosa after treatment by both routes, markedly lower IVM concentrations were recovered in the abomasal contents after its SC injection. While the active secretion of IVM from the bloodstream to the abomasal lumen is of little relevance [18], the adsorption of IVM to ruminal particulate material may account for its low oral bioavailability which was estimated in about 25\% [19].

High prevalence of anthelmintic resistance has now been reported in all parts of the world for GI helminth parasites, being nematodes of sheep and goats commonly involved [5,20,21]. In Uruguay, the development of anthelmintic resistance in sheep nematodes is not an exception. Resistance to IVM in sheep nematodes increased from 1.2\% [22] to 65\% [15] between 1996 and 2002. The trial described here demonstrated that current IVM resistance situation at the farm in which the study was conducted, is dramatically serious. The initially high IVM efficacy against GI nematodes in sheep has now drastically fell down, with almost a complete therapeutic failure to control some GI nematodes. Efficacies (evaluated by means the FECRT) as low as 7.1, 5.8 and $0 \%$ were observed for the RF, T1 and T2 IVM preparations under assay, respectively.

The identification of adult worms in the untreated lambs permitted to establish that the lambs were infected with Haemonchus spp., T.circumcincta, Trichostrongylus spp., Nematodirus spp., Cooperia spp., Oesophagostomum spp. and T.ovis. Nematode resistance in the current experiment was mainly related to Haemonchusspp., where all the IVM formulations failed to control this abomasal parasite. However, the clinical efficacy study also revealed a resistance-mediated failure to control $T$. circumcincta, where only efficacies $\leq 57 \%$ were observed. Resistance of T. circumcincta to IVM in Uruguay is reported here for the first time, which it may be useful as an indicator of the complexity of the resistance development phenomenon and its impact on livestock production.

All the tested IVM formulations also failed to control Cooperiaspp. and Nematodirus spp.. However, the low number of these parasites in the untreated control animals, limited the relevance of this finding. Contrarily, IVM demonstrated to maintain high efficacy against Trichostrongylus spp., Oesophagostomum spp. and T.ovis. Only Haemonchus spp. $\mathrm{L}_{3}$ larvae were recovered from the fecal cultures obtained from all the IVM treated 
groups. However, larvae obtained from fecal cultures are not necessarily related to parasites found at necropsies, since the high egg output observed in Haemonchus spp. may "mask" other nematodes.

Oppositely to what has been observed for other anthelmintics (such as the benzimidazole compounds), no significant differences on relative bioavailability/systemic exposure were observed among the tested IVM oral formulations in lambs. It is likely that any pharmaceutical/manufacturing change may more deeply affect the systemic availability of those compounds where GI absorption largely depends on the dissolution of low water soluble drug particles (suspension) in the abomasal lumen (i.e. albendazole), compared to the more lipophilic compounds such as IVM, but formulated as a mixed organic/aqueous solution. In spite of the fact that all the IVM formulations showed to reach an equivalent systemic exposure, all of them failed to control some common GI nematodes. The resistance status observed at the farm where the current trial was conducted is likely to be an indicator of the overall situation of the sheep flocks in Uruguay, and perhaps in many other regions of the world, where IVM completely failed to control H.contortus. This overall picture described in Uruguay, with resistance extended into other avermectin-type compounds, may be even worse if we consider that resistant $T$. circumcincta has been reported for the first time.

\section{Conclusions}

Neither the overall kinetic behaviour nor the IVM systemic exposure differed among all the tested oral formulations. Equivalent efficacy results were obtained for the different preparations, with an evident therapeutic failure to control Haemonchus spp. and T. circumcincta, which correlates with a high degree of nematode resistance to IVM.

\section{Methods}

\section{Chemicals}

Standards of IVM and abamectin (ABA), used as internal standard, were obtained from Sigma Chemical Company (Saint Louis, MO, USA). Three oral IVM formulations approved and commercially available to use in sheep in the pharmaceutical market in Uruguay, were used in the current experiment. The comparison included: Ivermectina $0.2 \mathrm{oral}^{\circledR}$ (IVM 0.2\%, Rosenbusch, Uruguay); Ivermic $0.2 \%{ }^{\circledR}$ (IVM 0.2\%, Microsules, Uruguay) and Ivomec ${ }^{\circledR}$ oral (IVM $0.08 \%$, Merial, The Netherlands). Ivomec ${ }^{\circledR}$ oral was considered the reference product (RF) as it was the pioneer first authorized product with a full dossier (NADA 131-392; approval date: July 26, 1988). The two IVM generic formulations were randomly designated as Test 1 (T1) and Test 2 (T2), respectively.

\section{Animals}

The study was conducted in a farm (Centro de Investigación y Experimentación "Dr. Alejandro Gallinal", Florida, Uruguay) where the failure of IVM to control GI nematodes had been previously demonstrated by the fecal egg counts reduction test (FECRT) [23]. One hundred and fifty six $(n=156)$ healthy male and female Corriedale lambs, not older than 1 year, weighing $29.5 \pm 5.6 \mathrm{~kg}$, body condition $3.1 \pm 0.6$, FAMACHA 1 [24] and naturally infected with GI nematodes, were involved in the trial. The criterion of inclusion for selection of the animals was based on worm egg per gram counts (epg) $(>200$ and $<8000 \mathrm{epg}$ ), body weight ( $\geq 20$ and $\leq 45 \mathrm{~kg}$ ), FAMACHA 1 and body condition $(\geq 2$ and $\leq 4)$ [25]. Throughout and 60 days before starting the experiment, animals grazed on a natural pasture and had free access to water. Animal procedures and management protocols were approved by the Ethics Committee according to the Animal Welfare Policy of the Faculty of Veterinary Medicine, Universidad de la República, Montevideo, Uruguay (http://www.fvet.edu.uy).

\section{Experimental design and treatments}

On day -1 , the experimental animals had an average of $2063 \pm 1635$ epg. The animals were ranked from lowest to highest epg counts. Based on increasing epg counts, replicates of 4 animals were formed. Within each replicate, animals were randomly assigned to treatment. The study was designed to have 39 animals per treatment group. One group of lambs was processed as the treated animals, but without drug treatment (untreated control). Animals in the other groups were treated with either the RF or each of the generic (T1 and T2) IVM formulations. All the IVM formulations were administered by the i.r. route at the dose rate of $0.2 \mathrm{mg} / \mathrm{kg}$ bodyweight. The i.r. route of administration was chosen in order to avoid leak/regurgitation of the administered dose and/or oesophageal grove closure, which commonly occurs after oral treatments affecting drug systemic availability [26]. Eight animals from each experimental group were randomly selected for the pharmacokinetic trial; being six of them used for the clinical efficacy trial. After selection, animals from the different groups involved in the pharmacokinetic and clinical efficacy trials have epg counts of $1489 \pm 252$.

\section{Sampling}

\section{Pharmacokinetic trial}

Heparinized blood samples $(5 \mathrm{~mL})$ were collected by jugular venipuncture prior to drug administration and at 1,3 , $6,12,18,24,36,48 \mathrm{~h}$, and $3,6,9,12$ and 15 days posttreatment. Blood samples were centrifuged at $3000 \times \mathrm{g}$ for $15 \mathrm{~min}$ and plasma was transferred to plastic tubes. All 
the plasma samples were stored at $-20^{\circ} \mathrm{C}$ until analyzed by high performance liquid chromatography (HPLC).

\section{Efficacy trial}

Individual fecal samples were collected from the rectum of each animal ( $\mathrm{n}=39$ each group) at 14 days posttreatment to assess the epg counts. Additionally, pooled samples were carried out in each experimental group for the coprocultures following the method described by Coles et al. [27]. Fourteen days after the treatment, six animals per experimental group were slaughtered for helminth recovery according of Veterinary Parasitology (WAAVP) guidelines [11]. The genera present in each GI compartment were identified and counted for each animal, separately, according the Ministry of Agriculture, Fisheries and Food [28], guidelines.

\section{IVM Analytical procedures}

\section{Sample clean-up and derivatization}

The extraction of IVM, from spiked and experimental plasma samples was carried out following the wellestablished technique [29]; slightly modified by 6 ). Aliquots of plasma $(1 \mathrm{~mL})$ sample was fortified with $20 \mu \mathrm{L}$ of ABA $(20 \mu \mathrm{g} / \mathrm{mL})$ (use as an internal standard) and acetonitrile $(1 \mathrm{~mL})$. Deionized water $(0.250 \mathrm{~mL})$ was added to each sample. The preparation was mixed using MultiTubevortexer (VWR Scientific Products, USA) for $20 \mathrm{~min}$ and the solvent-sample mixture was centrifuged at $2000 \mathrm{~g}$ during $10 \mathrm{~min}$. The supernatant was manually transferred into a tube. The supernatant was applied to a conditioned disposable C18 column (RP-18, $100 \mathrm{mg}$, Strata ${ }^{\circledR}$, Phenomenex, CA, USA), previously conditioned by passing $2 \mathrm{~mL}$ methanol and $2 \mathrm{~mL}$ deionized water. After washing with $1 \mathrm{~mL}$ of deionized water followed by $1 \mathrm{~mL}$ of water/methanol (4:1), the cartridges were dried for $5 \mathrm{~min}$ and the compounds were eluted with $1.5 \mathrm{~mL}$ of methanol and concentrated to dryness under a stream of nitrogen at $56^{\circ} \mathrm{C}$ in a water bath. The resuspension was carried out with $100 \mu \mathrm{L}$ of a solution of $\mathrm{N}$ methylimidazole (Sigma Chemical, St. Louis, MO, USA) in acetonitrile (1:1) [30]. Derivatization was initiated by adding $150 \mu \mathrm{L}$ of trifluoroacetic anhydride (Sigma Chemical, St Louis, MO, USA) solution in acetonitrile (1:2). After completion of the reaction $(<30 \mathrm{sec})$, an aliquot $(100 \mu \mathrm{L})$ of this solution was injected directly into the chromatograph.

\section{High performance liquid chromatography (HPLC) and validation}

IVM concentrations were determined by HPLC using a Shimadzu 10A HPLC system with autosampler (Shimadzu Corporation, Kyoto, Japan). HPLC analysis was undertaken using a reverse phase C18 column (Phenomenex, $5 \mu \mathrm{m}, 4.6 \mathrm{~mm} \times 250 \mathrm{~mm}$ ) and an acetic acid $0.2 \%$ in water methano $/$ acetonitrile (3.8/40 56.2) mobile phase at a flow rate of $1.5 \mathrm{~mL} / \mathrm{min}$ at $30^{\circ} \mathrm{C}$. IVM was detected using a fluorescence detector (Shimadzu, RF-10A Spectrofluorometric detector, Kyoto, Japan), readings at $365 \mathrm{~nm}$ (excitation wavelength) and $475 \mathrm{~nm}$ (emission wavelength). IVM concentrations were determined by the internal standard method using the Class LC 10 Software version 1.2 (Shimadzu Corporation, Kyoto, Japan) on an IBM compatible AT computer. The peak area ratios were considered to calculate the IVM concentrations in spiked (validation) and experimental plasma samples. There was no interference of endogenous compounds in the chromatographic determinations. The solvents (Baker, Phillipsburg, NJ, USA) used during the extraction and drug analysis were HPLC grade.

\section{Method validation}

A complete validation of the analytical procedures used for extraction and quantification of IVM was performed before starting analysis of the experimental samples obtained during the pharmacokinetic trial. Calibration curves in the range between $0.1-2.0 \mathrm{ng} / \mathrm{mL}$ and $2.0-40 \mathrm{ng} / \mathrm{mL}$ were plotted using the peak area ratios between analyte and the internal standard. Calibration curves were established using least squares linear regression analysis and correlation coefficients (r) and CV calculated. Linearity was established to determine the IVM concentrations/detector responses relationship. Percentages of IVM recovery from plasma were obtained in the range between 0.2 and $20 \mathrm{ng} / \mathrm{mL}$. The interassay precision of the extraction and chromatography procedures was estimated by processing replicate aliquots $(n=6)$ of pooled sheep plasma samples containing known IVM concentrations (0.1-2.0 and 2.0-40 ng/mL) on different working days. The $\mathrm{CV}$ for recovery and inter-day precision of the method were calculated. The limit of detection (LOD) was estimated according to the following equation [24]: $\mathrm{LOD}=\mathrm{A} / \mathrm{B}+(\mathrm{SD} * 3)$, where $\mathrm{A}$ is the baseline threshold at the retention time of each compound $(n=6)$ in spiked plasma samples, $B$ the peak area of the internal standard (ABA), and SD the standard deviation obtained from A. The limit of quantification (LOQ) was defined as the lowest measured concentration with a $\mathrm{CV}$ $<20 \%$ an accuracy of $\pm 20 \%$ and an absolute recovery $\geq 70 \%$. Concentration values below the LOQ were not considered for the kinetic analysis of experimental data.

\section{Pharmacokinetic analysis of the data}

Non-compartmental pharmacokinetic calculations for the concentration versus time curves for IVM in plasma for each individual animal after the different treatments were conducted using the $\mathrm{R}$ software (version 2.14.0). The peak concentration $\left(C_{\max }\right)$ and time to peak concentration $\left(\mathrm{T}_{\max }\right)$ were recorded directly from the measured concentration data. The elimination half-life $\left(T_{1 / 2 \mathrm{el}}\right)$ was 
calculated as $\ln 2 / \lambda_{\mathrm{el}}$, where the terminal elimination rate constant $\left(\lambda_{\mathrm{el}}\right)$, was calculated by performing regression analysis using data points belonging of the terminal phase concentration-time plot. The area under the plasma concentration-time curve from zero up to the limit of quantification $\left(\mathrm{AUC}_{0-\mathrm{LOQ}}\right)$ was calculated by means of the trapezoidal rule [31] and further extrapolated to infinity $\left(\mathrm{AUC}_{0-\infty}\right)$ by dividing the last experimental concentration by the terminal elimination rate constant $\left(\lambda_{\mathrm{el}}\right)$. Statistical moment theory was applied to calculate the mean residence time (MRT) by using the formula MRT = AUMC $\mathrm{AUC}_{0-\mathrm{LOQ}}$ [32] where AUMC is the area under the curve of the product of time and the plasma drug concentration vs. time from zero to infinity [31], and $\mathrm{AUC}_{0-\mathrm{LOQ}}$ is as defined above. $C_{\max } / A U C_{0-L O Q}$. was calculated by dividing the $\mathrm{C}_{\max }$ by $\mathrm{AUC}_{0-\mathrm{LOQ}}$. The distribution and elimination were calculated as plasma clearance per fraction of the dose absorbed $(\mathrm{CL} / \mathrm{F})$ calculated using $\mathrm{AUC}_{0-\mathrm{LOQ}}$ and apparent volume of distribution during the elimination phase per fraction of the dose absorbed $\left(\mathrm{Vd}_{\lambda \mathrm{el}} / \mathrm{F}\right)$. Relative bioavailability (F\%) was measured by comparing the $\mathrm{AUC}_{0-\mathrm{LOQ}}$ of the Test formulation with the $\mathrm{AUC}_{0-\mathrm{LOQ}}$ of the RF, using the following equation [33]:

$$
F \%=\mathrm{AUC}_{\text {test }} / \mathrm{AUC}_{\mathrm{RF}} * 100
$$

\section{Efficacy assessment}

The FECRT were calculated according to the method described in the WAAVP recommendations for detection of anthelmintic resistance [27]. The percentage of efficacy $(\% \mathrm{E})$ of each anthelmintic treatment against a given parasite species was determined by the comparison of worm burdens in treated (groups RF, T1 and T2) versus untreated control animals using the following formula [11]:\% $\mathrm{E}=$ (geometric mean of controls - geometric mean of treated/geometric mean of controls) $\times 100$. The genera and species of the third stage larvae recovered from faecal pool cultures or adult nematodes recovered from parasitized lambs (Groups RF, T1, T2 and untreated control) were identified following the Ministry of Agriculture, Fisheries and Food [28] guidelines.

\section{Statistical analysis of the data}

The pharmacokinetic parameters, concentration data, epg and nematode counts are reported as arithmetic mean \pm SD. Parametric (ANOVA + Tuckey) or non parametric (Kruskal-Wallis) test were used for the statistical comparison of the pharmacokinetic and efficacy data obtained from the different experimental groups. The assumption that the data obtained after treatments have the same variance was assessed. Prior to analysis, the individual epg and nematode counts were transformed using $\left(\log _{10+n}\right)$. A value of $P<0.05$ was considered statistically significant. The statistical analysis was performed using the R software, version 2.14.0 [34].

\section{Competing interests}

The authors declare that they have no competing interests.

\section{Authors' contributions}

GS conceived the study and participate in the animal and analytical phase of the experiment and in writing the draft manuscript. DC and LA conceived the study, participated in its design and in the animal phase and revised the draft version of the manuscript. CL conceived the study, participated in its design and revised the draft version of the manuscript. OC participated in the parasitological analysis and revised the draft version of the manuscript.PF participated in the pharmacological analysis and revised the draft version of the manuscript. All authors have read and approved the final manuscript.

\section{Acknowledgements}

This work was supported by Agencia Nacional de Innovación e Investigación (ANII) from Uruguay. The authors wish to thank the invaluable support of the people of the Secretariado Uruguayo de la Lana (SUL), for providing help in manage of the animals for this trial and to of Silvia Bentancor (Facultad Veterinaria, Universidad de la República, Uruguay), for providing help in efficacy trial.

\section{Author details}

${ }^{1}$ Área Farmacología, Facultad de Veterinaria, Universidad de la República, Montevideo, Uruguay. 'Laboratorio de Farmacología, Centro de Investigación Veterinaria de Tandil (CIVETAN), CONICET, Facultad de Ciencias Veterinarias, Universidad Nacional del Centro de la Provincia de Buenos Aires, Campus Universitario, (7000) Tandil, Argentina. ${ }^{3}$ Área de Investigación del Secretariado Uruguayo de la Lana (SUL), Florida, Uruguay. ${ }^{4}$ Laboratorio de Parasitología, Facultad de Veterinaria, Universidad de la República, Montevideo, Uruguay. ${ }^{5}$ Departamento de Ciencias Farmacéuticas, Facultad de Química, Universidad de la República, Montevideo, Uruguay.

Received: 16 July 2012 Accepted: 8 January 2013

Published: 11 February 2013

\section{References}

1. Benz GW, Roncalli RA, Gross S: Use of ivermectin in cattle, sheep, goats and swine. In Ivermectin and Abamectin. Edited by Campbell WC. New York: Springer; 1989:215-229.

2. Campbell WC, Benz GW: Ivermectin: a review of efficacy and safety. J Vet Pharmacol Ther 1984, 7:1-16.

3. McKellar QA, Benchaoui H: Avermectins and milbemycins. J Vet Pharmacol Ther 1996, 19:331-351.

4. Coop R, Barger I, Jackson F: The use of macrocyclic lactones to control parasites of sheep and goats. In Macrocyclic Lactones in Antiparasitic Therapy Edited by Versruysse J, Rew R. USA: CABI publishing New York; 2003:303-321.

5. Waller P: Global perspectives on nematode parasite control in ruminant livestock: the need to adopt alternatives to chemotherapy, with emphasis on biological control. Anim Health Res Rev 2003, 4:35-44.

6. Lifschitz A, Virkel G, Pis A, Imperiale F, Sanchez S, Alvarez L, Kujanek R, Lanusse $C$ : Ivermectin disposition kinetics after subcutaneous and intramuscular administration of an oil-based formulation to cattle. Vet Parasitol 1999, 86:203-215.

7. Lo PKA, Fink DW, Williams JB, Blodinger J: Pharmacokinetic studies of ivermectin: effects of formulation. Vet Res Commun 1985, 9:251-258.

8. Lifschitz A, Sallovitz J, Imperiale F, Pis A, Jaureguilorda J, Lanusse C: Pharmacokinetic evaluation of four ivermectin generic formulations in calves. Vet Parasitol 2004, 119:247-257.

9. Eslami A, Rassouli A, Meshki B, Shams GR: A bioequivalence study of an albendazole oral suspension produced in Iran and a reference product in Sheep. Int J Appl Res Vet Med 2006, 4:109-114.

10. Suarez G, Alvarez L, Castells D, Correa O, Fagiolino P, Lanusse C: Comparative drug systemic exposure and clinical efficacy against resistant nematodes in lambs treated with different albendazole formulations. J Vet Pharmacol Ther 2011, 34:557-564.

11. Wood I, Amaral N, Bairden K, Duncan J, Kassai T, Malone J: World Association for the Advancement of Veterinary Parasitology (W.A.A.V.P.) 
second edition of guidelines for evaluating the efficacy of anthelmintics in ruminants (bovine, ovine, caprine). Vet Parasitol 1995, 58:181-213.

12. Baggot J, McKellar Q: The absorption, distribution and elimination of anthelmintic drugs: the role of pharmacokinetics. J Vet Pharmacol Ther 1994, 17:409-419.

13. McKellar Q, Gokbulut C: Pharmacokinetic features of the antiparasitic macrocyclic lactones. Curr Pharm Biotechnol 2012, 13:888-911.

14. Toutain P, Koritz G: Veterinary drug bioequivalence determination. J Vet Pharmacol Ther 1997, 20:79-90.

15. Lloberas M, Alvarez L, Entrocasso C, Virkel G, Lanusse C, Lifschitz A: Measurement of ivermectin concentrations in target worms and host gastrointestinal tissues: influence of the route of administration on the activity against resistant Haemonchus contortus in lambs. Exp Parasitol 2012, 131:304-309.

16. Marriner SE, McKinnon I, Bogan JA: The pharmacokinetics of ivermectina after oral and subcutaneous administration to sheep and horses. J Vet Pharmacol Ther 1987, 1:175-179.

17. Imperiale F, Lifschitz A, Sallovitz J, Virkel G, Lanusse C: Comparative depletion of ivermectin and moxidectin milk residues in dairy sheep after oral and subcutaneous administration. J Dairy Res 2004, 71:427-433.

18. Hennessy DR, Page SW, Gottschall D: The behaviour of doramectin in the gastrointestinal tract, its secretion in bile and pharmacokinetic disposition in the peripheral circulation after oral and intravenous administration to sheep. J Vet Pharmacol Ther 2000, 23:203-213.

19. Prichard RK, Steel JW, Lacey E, Hennessy DR: Pharmacokinetics of ivermectin in sheep following intravenous, intra-abomasal or intraruminal administration. J Vet Pharmacol Therap 1985, 8:88-94.

20. Wolstenholme A, Fairweather I, Prichard R, Samson-Himmelstjerna G, Sangster N: Drug resistance in veterinary helminths. Trends Parasitol 2004, 20:469-476.

21. Kaplan R: Drug resistance in nematodes of veterinary importance: a status report. Trends Parasitol 2004, 20:477-481.

22. Nari A, Salles J, Gil A, Waller P, Hansen J: The prevalence of anthelmintic resistance in nematode parasites of sheep in Southern Latin America: Uruguay. Vet Parasitol 1996, 62:213-222.

23. Castells D, Mederos A, Lorenzelli E, Macchi I: Diagnosticos de resistencia antihelmíntica de Haemonchus spp. a las ivermectinas en el Uruguay. In Resistencia genética del ovino y su aplicación en sistemas de control integrado de parásitos. Edited by FAO. Rome (Italy): Animal Production and Health Paper (FAO) / FAO; 2002:61-66.

24. Van Wyk J, Bath G: The FAMACHA ${ }^{\circledR}$ system for managing haemonchosis in sheep and goats by clinically identifying individual animals for treatment. Vet Res 2002, 33:509-529.

25. Russel A: Body condition scoring of sheep. In Sheep and goat practice. Edited by Boden E. London: BailliereTindall; 1991:3-10.

26. Prichard RK, Steel JW, Lacey E, Hennessy DR: Pharmacokinetics of ivermectin in sheep following intravenous, intra-abomasal or intraruminal administration. J Vet Pharmacol Ther 1985, 8:88-94.

27. Coles G, Bauer C, Borgsteede F, Geerts S, Klei T, Taylor M, Waller P: World association for the advancement of veterinary parasitology (WAAVP) methods for the detection of anthelmintic resistance in nematodes of veterinary importance. Vet Parasitol 1992, 44:35-44.

28. Ministry of Agriculture, Fisheries and Food: Manual of Veterinary Parasitological Laboratory Techniques. London: HMSO; 1986:1-152.

29. Alvinerie M, Sutra JF, Galtier P: Ivermectin in goat milk after subcutaneous injection. Vet Res 1993, 24:417-421.

30. De Montigny P, Shim J, Pivinichny J: Liquid chromatographic determination of ivermectin with trifluoro-acetic anhydride and N-methylimidazole as the derivatization reagent. J Pharmaceut Biomed Anal 1990, 8:507-511.

31. Gibaldi M, Perrier D: Pharmacokinetics. 2nd edition. New York, USA: Marcel Dekker; 1982:45-109. revised and expanded.

32. Perrier D, Mayersohn M: Non-compartmental determination of the steady-state volume of distribution for any mode of administration. J Pharm Sci 1982, 71:372-373.

33. Toutain P, Bousquet-Mélou A: Bioavailability and its assessment. J Vet Pharmacol Therap 2004, 27:455-466.

34. R Development Core Team: R: A language and environment for statistical computing. In R Foundation for Statistical Computing. Vienna, Austria; 2012.

doi:10.1186/1746-6148-9-27

Cite this article as: Suárez et al:: Relative bioavailability and comparative clinical efficacy of different ivermectin oral formulations in lambs. BMC Veterinary Research 2013 9:27.

\section{Submit your next manuscript to BioMed Central and take full advantage of:}

- Convenient online submission

- Thorough peer review

- No space constraints or color figure charges

- Immediate publication on acceptance

- Inclusion in PubMed, CAS, Scopus and Google Scholar

- Research which is freely available for redistribution 